\title{
USING AHP TO SELECT PROJECTS IN URBAN PLANNING
}

\author{
K. Anagnostopoulos, M. Giannopoulou, Y. Roukounis, A. Vavatsikos \\ Dpt. of Civil Engineering \\ Democritus University of Thrace \\ Vassilissis Sofias 1, 67100 Xanthi - Greece \\ yrouk@civil.duth.gr
}

Keywords: urban planning, project evaluation

Summary: The AHP was used to evaluate projects proposed by a development study concerning a medium size city in Greece. The method was proved to be a persuasive response in a new context characterized by large changes in the administrative level and in the socio-economic environment of the region. The problem was modeled on two hierarchy levels and the sensitivity analysis was showed a remarkable stability in the final rating of the projects. Experts in urban planning appreciated the method as a tool for selecting projects.

\begin{abstract}
The new role of the Greek cities is determined by both the Development Plan of the Community Space and the new parameters (administrative restructuring at local level, competencies at higher administrative levels), which come into play and impose a multilevel approach to urban planning. Local Administration Organizations hence extend over geographical areas of substantial surface, disposing important population basis, institutional and financial planning tools with possibilities of mobilizing private funds and important spatial planning capacities. Furthermore, the higher administration levels are reset and redefined on the basis of new competencies in the framework of administrative decentralization as well as broader administrative modernization. The goals and orientations of the development strategy for the cities connect the developmental process at the specific level with supra-structure developmental plans (the inter-community one, the prefectorial, regional and national ones) and are specialised accordingly, in relation to the local conditions, problems and potential of each area.

We have use the AHP in order to rationalize the selection of projects in a medium size city. The application of the method was based on the interventions proposed in a study for the Municipality of Orestiada, focusing on 35 of these interventions directly related with the development of the city (Development Strategy Study, 2000). Orestiada is the second largest urban centre of the Evros Prefecture, an area stretching at the NE part of the country on the borders with Turkey and Bulgaria. The city is strongly interdependent with and influenced by the adjacent communities, at various levels and varying intensity, as well as other areas of the Evros prefecture at large. These relations were shaped and evolved gradually, through the 76-year life of the city, on geographical, operational, historical and social terms. The city has currently prospects of development within a new framework, which can strengthen its importance in the region and its spatial role as an urban centre, due to its geographical position in the prefecture and the region as well as the economic evolutions in its area of direct impact and the overall new conditions shaping the neighbouring international space.
\end{abstract}

The strategy for the Orestiada city is placed within this framework and sets the following strategic goals: a/ The strengthening of its role as a hub. b/ Its transformation into a development centre of trans-national scope. $\mathrm{c} /$ The strengthening of its attraction, extrovert character and competitiveness. $\mathrm{d} / \mathrm{The}$ enhancement of its business infrastructure. These goals lead to a series of interventions, 35 of which are selected to be evaluated. The interventions chosen are distributed in six groups which are formed according to a uni- 
form sectoral rationale following the general principles on which the Region of Eastern Macedonia and Trace structures the Regional Operational Plan and the central administration services elaborate relative studies and draw up the Regional Development Plan (Regional Operational Plan, 1999): productive infrastructure (8), technical infrastructure (10), social infrastructure (8), revival of the city's cultural face (3), natural environment and leisure (1), regulatory and urban planning (5).

The problem is structured on two hierarchies. On the 1 st level of the first hierarchy the goal set is the overall development of Orestiada. On the 2nd level the main subgoals are set which, as demonstrated by the study, contribute decisively to the development of the city: a/ Promotion of the city as an international trade and transportation hub. b/ Exploitation and sustainable use of natural resources. c/ Exploitation, development and expansion of the industrial basis. $\mathrm{d} /$ Treatment of the demographic shrinkage problem. e/ Smoothing of the intra-regional disparities. f/ Productivity increases in the primary and secondary sectors. The 3rd level of the hierarchy consists of the economic, social and environmental criteria. On the 4th level of the hierarchy the subcriteria of the 3rd level criteria are placed, with a view to presenting a more detailed description of the problem parameters. The last level of the hierarchy consists of the alternative options, the specific interventions which are directed towards the sectors of the productive, technical and social infrastructure, the revival of the city's cultural physiognomy, natural environment and leisure and, finally, the spatial and urban planning. On the second hierarchy, the evaluation is based on the contribution of each project to the various intervention sectors, as described and assessed at the first hierarchy. On the first level, the goal set is the optimal ranking of the proposed projects. The second level is shaped by the intervention sectors, which constitute the basic criteria for the evaluation of the proposed projects. The cost criteria of the proposed projects and their funding possibility, which are important parameters to be taken into account when setting the priority between those projects, are also placed on the same level. On the 3rd level the elements placed are each project's contribution intensities to the satisfaction of each criterion.

The final project priorities are noteworthy. The projects ranking in the first 15 positions regard: i/ The basic infrastructure for the improvement of the urban environment, through the construction of new or the amelioration of already existing infrastructures (internal road network, sewage system, public health). ii/ The improvement of knowledge on issues related to the latest evolutions in the primary and the secondary sectors of the economy. iii/ The strengthening of the infrastructure supporting venturing and innovative spirit, and the processing and exchange of productive commodities, as well as the exploitation and trade of local plant and animal production, recycling and exploiting by-products, etc. It is characteristic that these projects remain first priority even when the criteria of "cost" and "financing possibility" are not included in the model. The high priority of these projects in the objective claims of the area and the satisfactory financing possibility are the elements attributing these projects their rank of priority. In the remaining positions, there is an intermingling of the projects pertaining to different intervention sectors, the unique exception being the projects referring to the cultural face of the city.

It is also very interesting to note the reactions caused by the use of AHP in a context where empirical rules and political considerations traditionally dominate the selection of projects. First, the final ranking was in accord with the «intuitive» evaluation of projects that experts in regional planning have proposed. Second, the local authorities despite their initial mistrust have accepted the method very well. Of course, the final decisions depend on others parameters, including pressures of local groups and political considerations, but we strongly believe that the final ranking will remain the background for the choices.

\section{References}

Development Strategy Study for the Orestiada Municipality (2000), Institute for Regional Development, Final Report, Athens.

Regional Operational Plan of Est Macedonia and Thrace Region (1999), Final Report, Komotini. 